\title{
Optimization on the distribution of population densities and the arrangement of urban activities
}

\author{
Savin Treanţă* \\ Department of Applied Mathematics, Faculty of Applied Sciences, University Politehnica of Bucharest, Romania
}

\begin{abstract}
In this paper, an approximation on the distribution of population densities and the arrangement of urban activities, over a set of $n$ locations, is derived by using the classical multiobjective optimization theory and Shannon entropy.
\end{abstract}

Keywords Weighted entropy; Utility distribution; Probability distribution; Efficiency conditions.

AMS 2010 subject classifications 94A17,46N10

DOI: $10.19139 /$ soic.v6i2.348

\section{Introduction, problem description and preliminaries}

The term of entropy was used for the first time in 1865 in Thermodynamics by Rudolf Clausius [7]. Later, scientists such as Ludwig Boltzmann, Josiah Willard Gibbs and James Clerk Maxwell gave to this concept a statistical basis. In Probability Theory, the degree of uncertainty to a random variable can also be evaluated using the entropy. Consequently, the entropy can be used in the study of some risk assessment problems arising in different fields.

The purpose of this paper is to develop, using the classical multiobjective optimization theory, a simultaneous optimization model involving Shannon entropy and spatial Shannon entropy subject to appropriate and meaningful constraints. Moreover, by considering the qualitative concept of utility, we extend our model to the case of BelişGuiaşu entropy and spatial Beliş-Guiaşu entropy. Practically, in both cases, we derive an approximation on the distribution of population densities and the arrangement of urban activities over a set of $n$ locations.

Now, let us introduce our study problem. According to Batty [4], following Batty [3], we shall represent a city as a set of locations. Also, we assume that: (1) there are $n$ locations, identified by $i$, with $i=1, \ldots, n$; (2) each location is a point or an area where urban activities can take place; (3) in each location there exists a number of units of urban activity; (4) the location identified by $i$ has the size (area) $a_{i}$ and, therefore, $A=\sum_{i=1}^{n} a_{i}$ is the total size (area) of the city. Denote by

$$
N=\sum_{i=1}^{n} N_{i}
$$

the total number of units of urban activity (the total amount of urban activity), where $N_{i}$, represents the number of units of urban acitiviy associated with location $i, i=\overline{1, n}$. If we start with $N_{1}$, the number of allocations of $N_{1}$ (i.e., the number of locations with $N_{1}$ units of urban activity) if given by

$$
C_{N}^{N_{1}}=\frac{N !}{N_{1} !\left(N-N_{1}\right) !},
$$

\footnotetext{
*Correspondence to: Department of Applied Mathematics, Faculty of Applied Sciences, University Politehnica of Bucharest, 313 Splaiul Independentei, 060042 - Bucharest, Romania. E-mail:savin_treanta@yahoo.com
}

ISSN 2310-5070 (online) ISSN 2311-004X (print)

Copyright (C) 2018 International Academic Press 
the number of locations with $N_{2}$ units of urban activity if given by

$$
C_{N-N_{1}}^{N_{2}}=\frac{\left(N-N_{1}\right) !}{N_{2} !\left(N-N_{1}-N_{2}\right) !}
$$

and so on. Making the product

$$
C_{N}^{N_{1}} \cdot C_{N-N_{1}}^{N_{2}} \cdot \ldots \cdot C_{N-N_{1}-\ldots-N_{n-1}}^{N_{n}}
$$

we find the total number of arrangements

$$
W=\frac{N !}{\prod_{i=1}^{n} N_{i} !},
$$

considered as a measure of complexity ( $W$ depends by allocations) of the city.

\section{Remark 1.1}

i) If the total amount of urban activity $N$ is allocated to $N_{i}$, with $i \in\{1, \ldots, n\}$ fixed, then the measure of complexity $W$ is equal to 1 .

ii) If $N_{i}=\frac{N}{n}, i=\overline{1, n}$, then $W$ varies with respect to the total amount of urban activity $N$ and the number of locations $n$.

By maximizing the measure of complexity $W$ (more precisely, the logarithm of $W$ ), we shall find the most enjoyable arrangement of units of urban activity in that it would provide the greatest possibility of distinct individual activities associated with the locations $i$. Usually, such maximizations are subject to appropriate and meaningful constraints. By a direct computation, using Stirling's formula, we get

$$
\ln W \approx N+\ln (N !)-\sum_{i=1}^{n} N_{i} \ln N_{i}
$$

Taking into account that $N_{i}$ is a frequency that can be trasformed into a probability $p_{i}=\frac{N_{i}}{N}$, by substituting the number of units of urban acitiviy associated with location $i$ in the previous relation (6) and dropping the constant terms, we find that the number of arrangements $W$ is proportional to Shannon entropy (measure of uncertainty, Shannon [14])

$$
H=-\sum_{i=1}^{n} p_{i} \ln p_{i}
$$

Consequently, to maximize $\ln W$ is equivalent with the well-known process of maximizing $H$.

Remark 1.2

i) When the total amount of urban activity $N$ is equally distributed to locations, that is $N_{i}=\frac{N}{n}, i=\overline{1, n}$, then $p_{i}=\frac{1}{n}$ and $H=\ln n$ is at a maximum. Also, let us remark that $H$ varies with $n$.

ii) If the total amount of urban activity $N$ is allocated to $N_{i}, i \in\{1, \ldots, n\}$ fixed, that is $N=N_{i}, i \in\{1, \ldots, n\}$ fixed, then $p_{i}=1$ and $p_{j}=0, j \in\{1, \ldots, n\}, i \neq j$, and $H=0$ is at a minimum.

Further, we consider the spatial entropy (for more details, the reader is directed to Batty [3], Batty et al. [5])

$$
S=-\sum_{i=1}^{n} p_{i} \ln \frac{p_{i}}{A_{i}}
$$

which takes into account the numbers $A_{i}=\frac{a_{i}}{A}$, where $a_{i}$ and $A$ are introduced at the beginning of this section. Let us notice that $\sum_{i=1}^{n} A_{i}=1$ and assume that $\frac{p_{i}}{A_{i}}$ is subunitary (otherwise, we must minimize $S$ instead of maximize it). 
Considering the previous mathematical context, the main aim of this paper is to study the following vector (bi-objective) optimization problem

$$
\begin{gathered}
(V O P) \quad \max _{p_{i}}\left(-\sum_{i=1}^{n} p_{i} \ln p_{i}, \quad-\sum_{i=1}^{n} p_{i} \ln \frac{p_{i}}{A_{i}}\right) \\
\text { subject to } \\
\sum_{i=1}^{n} p_{i}=1 \\
\sum_{i=1}^{n} p_{i} P_{i}=\bar{P} \\
\sum_{i=1}^{n} p_{i} c_{i}=\bar{C} \\
\sum_{i=1}^{n} p_{i} \ln a_{i}=\bar{A},
\end{gathered}
$$

where $p_{i}$ is the probability of finding a place $i$ which has $P_{i}$ population residing there and $c_{i}$ the travel cost from the central business district to the zone $i$. The constraint (10) is a normalization constraint on the probabilities, (11) is a constraint on the mean population of places, (12) is a constraint on the average travel cost incurred by population and, finally, (13) is a constraint on the average "logarithmic" size of locations.

The second objective of this work is to investigate a similar problem which involves the qualitative concept of utility. The models proposed here can be regarded as an approximation on (i) the distribution of population densities and (ii) the arrangement of urban activities over a set of $n$ locations.

Next, in order to develop our theory, we will enunciate some elements of multiobjective optimization. Consider the following convention between two vectors, $u=\left(u_{1}, \ldots, u_{s}\right), v=\left(v_{1}, \ldots, v_{s}\right)$ in $R^{s}$

$$
\begin{gathered}
u=v \Leftrightarrow u_{i}=v_{i}, \quad u \leq v \Leftrightarrow u_{i} \leq v_{i}, \\
u<v \Leftrightarrow u_{i}<v_{i}, \quad u \preceq v \Leftrightarrow u \leq v, u \neq v, \quad i=\overline{1, s},
\end{gathered}
$$

and the following vector minimization problem

$$
(P) \quad \min f(x)
$$

subject to

$$
x \in X:=\left\{x \in R^{n} \mid g(x) \leq 0\right\},
$$

where $f: R^{n} \rightarrow R^{s}$ and $g: R^{n} \rightarrow R^{m}$ are vector-valued functions, defined by $f(x)=\left(f_{1}(x), f_{2}(x), \ldots, f_{s}(x)\right)$ and $g(x)=\left(g_{1}(x), g_{2}(x), \ldots, g_{m}(x)\right)$, with $f_{i}: R^{n} \rightarrow R, i \in\{1, \ldots, s\}$, and $g_{j}: R^{n} \rightarrow R, j \in\{1, \ldots, m\}$, continuously differentiable functions on $R^{n}$. Denote by $\nabla f_{i}(x)$ and $\nabla g_{j}(x)$ the gradients of $f_{i}$ and $g_{j}$ at $x \in R^{n}$, respectively, and by $\langle x, y\rangle=\sum_{i=1}^{s} x_{i} y_{i}$ the inner product of $x \in R^{s}$ and $y \in R^{s}$.

Definition 1.1

A point $x^{0} \in X$ is said to be an efficient solution to problem $(P)$ if there is no $x \in X$ such that $f(x) \preceq f\left(x^{0}\right)$. 


\section{Definition 1.2}

A point $x^{0} \in X$ is said to be a weak efficient solution to problem $(P)$ if there is no $x \in X$ such that $f(x)<f\left(x^{0}\right)$.

Obviously, if $x^{0} \in X$ is an efficient solution to problem $(P)$ then $x^{0}$ is a weak efficient solution to problem $(P)$. However, the converse relation does not hold, in general, and practically the concept of efficient solution is more desirable than that of weak efficient solution.

Theorem 1.1

(Necessary efficiency conditions for $(P)$ ) Let $x^{0} \in X$ be any feasible solution to $(P)$ and suppose that the generalized Guignard constraint qualification holds at $x^{0} \in X$. If $x^{0} \in X$ is an efficient solution to $(P)$, then there exist the vectors $\lambda \in R^{s}$ and $\mu \in R^{m}$ such that

$$
\begin{gathered}
\sum_{i=1}^{s} \lambda_{i} \nabla f_{i}\left(x^{0}\right)+\sum_{j=1}^{m} \mu_{j} \nabla g_{j}\left(x^{0}\right)=0 \\
\left\langle\mu, g\left(x^{0}\right)\right\rangle=0 \\
\lambda>0, \quad \mu \geq 0 .
\end{gathered}
$$

\section{Remark 1.3}

If the vector minimization problem $(P)$ contains, in addition, constraints of the type $h(x)=0$, with $h: R^{n} \rightarrow R^{l}$ a continuously differentiable function, then there exists a vector $\alpha \in R^{l}$ such that the first condition in (16) becomes

$$
\sum_{i=1}^{s} \lambda_{i} \nabla f_{i}\left(x^{0}\right)+\sum_{k=1}^{l} \alpha_{k} \nabla h_{k}\left(x^{0}\right)+\sum_{j=1}^{m} \mu_{j} \nabla g_{j}\left(x^{0}\right)=0 .
$$

Let $\rho$ be a real number, $C \subseteq R^{n}$, and $b: C \times C \rightarrow[0, \infty)$ a function.

Definition 1.3

A differentiable function $\phi: C \rightarrow R$ is said to be [strictly] $(\rho, b)$-quasiinvex at $x^{0} \in C$ with respect to $\eta$ and $\theta$ if there exist the vector functions $\eta: C \times C \rightarrow R^{n}$ and $\theta: C \times C \rightarrow R^{q}$ such that for any $x \in C,\left[x \neq x^{0}\right]$, the following implication holds

$$
\phi(x) \leq \phi\left(x^{0}\right) \Longrightarrow b\left(x, x^{0}\right) \eta\left(x, x^{0}\right) \nabla \phi\left(x^{0}\right)[<] \leq-\rho b\left(x, x^{0}\right)\left\|\theta\left(x, x^{0}\right)\right\|^{2} .
$$

In the above definition, if we replace " $\leq "$ with " = ", we obtain the definition of monotonic $(\rho, b)$-quasiinvexity at $x^{0}$ with respect to $\eta$ and $\theta$.

In the following theorem, we establish, under $(\rho, b)$-quasiinvexity assumptions, sufficient optimality conditions for $(P)$.

\section{Theorem 1.2}

(Sufficient efficiency conditions for $(P)$ ) Let $x^{0} \in X$ be any feasible solution to $(P)$ and let there exist the vectors $\lambda \in R^{s}$ and $\mu \in R^{m}$ such that the conditions (16) are satisfied. If:

(i) each function $f_{i}(x), i=\overline{1, s}$, is $\left(\rho_{i}^{1}, b\right)$-quasiinvex at $x^{0}$ with respect to $\eta$ and $\theta$ and there exists at least an index $k \in\{1, \ldots, s\}$ such that $f_{k}(x)$ is strictly $\left(\rho_{k}^{1}, b\right)$-quasiinvex at $x^{0}$ with respect to $\eta$ and $\theta$;

(ii) each function $g_{j}(x), j=\overline{1, m}$, is monotonic $\left(\rho_{j}^{2}, b\right)$-quasiinvex at $x^{0}$ with respect to $\eta$ and $\theta$;

(iii) $\sum_{i=1}^{s} \lambda_{i} \rho_{i}^{1}+\sum_{j=1}^{m} \mu_{j} \rho_{j}^{2} \geq 0$

then $x^{0} \in X$ is an efficient solution to $(P)$. 


\section{Remark 1.4}

If the vector minimization problem $(P)$ contains, in addition, constraints of the type $h(x)=0$, with $h: R^{n} \rightarrow R^{l}$ a continuously differentiable function, then the conditions $(i)$ and (iii) from Theorem 1.2 change as follows:

( $\left.i^{\prime}\right)$ each function $f_{i}(x), i=\overline{1, s}$, is $\left(\rho_{i}^{1}, b\right)$-quasiinvex at $x^{0}$ with respect to $\eta$ and $\theta$;

( $i$ ”) each function $h_{k}(x), k=\overline{1, l}$, is $\left(\rho_{k}^{3}, b\right)$-quasiinvex at $x^{0}$ with respect to $\eta$ and $\theta$;

$\left(i\right.$ '”) one of the functions given in $\left(i\right.$ '), $\left(i\right.$ ') is strictly $(\rho, b)$-quasiinvex at $x^{0}$ with respect to $\eta$ and $\theta$, where $\rho=\rho_{i}^{1}$ or $\rho_{k}^{3}$,

and, respectively

$$
\text { (iii') } \sum_{i=1}^{s} \lambda_{i} \rho_{i}^{1}+\sum_{j=1}^{m} \mu_{j} \rho_{j}^{2}+\sum_{k=1}^{l} \alpha_{k} \rho_{k}^{3} \geq 0 \text {. }
$$

For more details, other notions and their connections, the reader is addressed to Yu [20], Treanţă and Udrişte [16], Arana et al. [2], Verma [18], Treanţă [17].

\section{Main results}

Let us observe that our bi-objective optimization problem (9), subject to (10) - (13), can be rewritten as follows

$$
\begin{gathered}
(V O P) \quad \min _{p_{i}}\left(\sum_{i=1}^{n} p_{i} \ln p_{i}, \quad \sum_{i=1}^{n} p_{i} \ln p_{i}-\sum_{i=1}^{n} p_{i} \ln A_{i}\right) \\
\text { subject to } \\
\sum_{i=1}^{n} p_{i}=1, \quad \sum_{i=1}^{n} p_{i} P_{i}=\bar{P}, \quad \sum_{i=1}^{n} p_{i} c_{i}=\bar{C}, \quad \sum_{i=1}^{n} p_{i} \ln a_{i}=\bar{A} .
\end{gathered}
$$

Taking into account the general context formulated in the previous section (see Theorem 1.1 and Remark 1.3), now we are in a position to establish and prove the first part of our main results.

\section{Theorem 2.1}

If $p=\left(p_{i}\right), i=\overline{1, n}$, is a normal efficient solution in $(V O P)$, then there exist the scalars $\lambda_{1}, \lambda_{2}, \alpha, \beta, \gamma, \delta$ with $\lambda_{1}>0, \lambda_{2}>0, \lambda_{1}+\lambda_{2}=1$, satisfying

$$
p_{i}=\exp \left[-1-\alpha-\left(1-\lambda_{1}\right) \ln A-\beta P_{i}-\gamma c_{i}-\left(\lambda_{1}+\delta-1\right) \ln a_{i}\right],
$$

or, equivalently,

$$
p_{i}=K \exp \left[-\beta P_{i}-\gamma c_{i}-\left(\lambda_{1}+\delta-1\right) \ln a_{i}\right],
$$

where $K=\exp \left[-1-\alpha-\left(1-\lambda_{1}\right) \ln A\right]$ is a constant of proportionality which ensures that the probabilities sum is 1. Moreover, the "negative" measures of complexity $H_{1}=-H=\sum_{i=1}^{n} p_{i} \ln p_{i}$ and $S_{1}=-S=\sum_{i=1}^{n} p_{i} \ln \frac{p_{i}}{A_{i}}$, which are at a minimum for the given set of constraints, simplify to

$$
\begin{gathered}
H_{1}=-1-\alpha-\left(1-\lambda_{1}\right) \ln A-\beta \bar{P}-\gamma \bar{C}-\left(\lambda_{1}+\delta-1\right) \bar{A} \\
S_{1}=-1-\alpha+\lambda_{1} \ln A-\beta \bar{P}-\gamma \bar{C}-\left(\lambda_{1}+\delta\right) \bar{A} .
\end{gathered}
$$




\section{Proof}

Having in mind the general mathematical framework formulated in Theorem 1.1 and Remark 1.3 of section 1 , we introduce the Lagrangian

$$
\begin{gathered}
L\left(p_{i} ; \lambda_{1}, \alpha, \beta, \gamma, \delta\right)=\sum_{i=1}^{n} p_{i} \ln p_{i}+\left(\lambda_{1}-1\right) \sum_{i=1}^{n} p_{i} \ln A_{i}+\alpha\left(\sum_{i=1}^{n} p_{i}-1\right) \\
+\beta\left(\sum_{i=1}^{n} p_{i} P_{i}-\bar{P}\right)+\gamma\left(\sum_{i=1}^{n} p_{i} c_{i}-\bar{C}\right)+\delta\left(\sum_{i=1}^{n} p_{i} \ln a_{i}-\bar{A}\right)
\end{gathered}
$$

and, by imposing the necessary conditions of efficiency, we get

$$
1+\ln p_{i}+\left(\lambda_{1}-1\right) \ln A_{i}+\alpha+\beta P_{i}+\gamma c_{i}+\delta \ln a_{i}=0,
$$

or, equivalently,

$$
\begin{gathered}
p_{i}=\exp \left[-1-\alpha-\left(1-\lambda_{1}\right) \ln A-\beta P_{i}-\gamma c_{i}-\left(\lambda_{1}+\delta-1\right) \ln a_{i}\right] \\
=K \exp \left[-\beta P_{i}-\gamma c_{i}-\left(\lambda_{1}+\delta-1\right) \ln a_{i}\right],
\end{gathered}
$$

where $K=\exp \left[-1-\alpha-\left(1-\lambda_{1}\right) \ln A\right]$ is a constant of proportionality which ensures that the probabilities sum is 1.

If we substitute the probability in (19) into the "negative" Shannon entropy $H_{1}=\sum_{i=1}^{n} p_{i} \ln p_{i}$ and into the "negative" spatial Shannon entropy $S_{1}=\sum_{i=1}^{n} p_{i} \ln \frac{p_{i}}{A_{i}}$, by a direct computation, we obtain the "negative" measures of complexity in (20) and the proof is complete.

Over the past years, in order to correlate the quantitative concept of information with the qualitative concept of utility, many researchers (see, for instance, Beliş and Guiaşu [6], Longo [12], Kapur [9], [10]) have introduced several weighted information measures. Given the context in which we are, these weighted measures of information become very important (they take into account both the probabilities with which certain random events occur and, also, some qualitative characteristics of these events). Thus, according to Beliş and Guiaşu [6], let $u_{i}$ be the weight associated to an elementary event with probability $p_{i}$ (in our case, an elementary event is the finding of a place $i$ which has $P_{i}$ population residing there and $c_{i}$ the travel cost from the central business district to the zone $i$ ). Consider the weight $u_{i}$ as a finite, positive real number representing the relevance, the significance or the utility of the occurrence of an event with probability $p_{i}$. If $u_{i}>u_{j}$, then the event with weight $u_{i}$ (and probability $p_{i}$ ) is strictly more significant, more useful or more relevant than the event with weight $u_{j}$ (and probability $p_{j}$ ), where $i, j \in\{1, \ldots, n\}, i \neq j$.

Using the previous utilities (weights), let us introduce the following weighted bi-objective optimization problem

$$
(V O P)^{*} \quad \min _{p_{i}}\left(\sum_{i=1}^{n} u_{i} p_{i} \ln p_{i}, \quad \sum_{i=1}^{n} u_{i} p_{i} \ln p_{i}-\sum_{i=1}^{n} u_{i} p_{i} \ln A_{i}\right)
$$

subject to

$$
\begin{gathered}
\sum_{i=1}^{n} p_{i}=1, \quad \sum_{i=1}^{n} u_{i} p_{i}=\tilde{P}, \quad \sum_{i=1}^{n} p_{i} P_{i}=\bar{P}, \\
\sum_{i=1}^{n} p_{i} \ln a_{i}=\bar{A}, \quad \sum_{i=1}^{n} u_{i} p_{i} \ln a_{i}=\tilde{A}, \quad \sum_{i=1}^{n} p_{i} c_{i}=\bar{C} .
\end{gathered}
$$




\section{Remark 2.1}

i) The above minimum is computed for fixed utility distributions.

ii) There are two additional constraints in $(V O P)^{*}$ compared to $(V O P)$ : the constraint $\sum_{i=1}^{n} u_{i} p_{i}=\tilde{P}$ on the relevance of the weights $u_{i}$ (of course, if $u_{1}=u_{2}=\cdots=u_{n}:=u$, we get $\tilde{P}=u$ and further, if $u=1$, we find the first constraint in $(V O P)^{*}$; therefore, for generality, we shall consider the weights $u_{i}$ as different, finite, positive real numbers) and the constraint $\sum_{i=1}^{n} u_{i} p_{i} \ln a_{i}=\tilde{A}$ on the weighted average "logarithmic" size of locations.

Now, we shall formulate and prove the second part of our main results.

\section{Theorem 2.2}

If $p=\left(p_{i}\right), i=\overline{1, n}$, is a normal efficient solution in $(V O P)^{*}$, then there exist the scalars $\lambda_{1}, \lambda_{2}, \alpha, \beta, \gamma, \delta, \theta$ and $\tau$, with $\lambda_{1}, \lambda_{2}>0, \lambda_{1}+\lambda_{2}=1$, satisfying

$$
p_{i}=\exp \left[-u_{i}-u_{i}\left(\lambda_{1}-1+\theta\right) \ln a_{i}-u_{i}\left(1-\lambda_{1}\right) \ln A-\alpha-\beta u_{i}-\gamma P_{i}-\delta \ln a_{i}-\tau c_{i}\right] / u_{i} .
$$

Moreover, the "negative" measures of complexity $H_{1}^{*}=\sum_{i=1}^{n} u_{i} p_{i} \ln p_{i}$ and $S_{1}^{*}=\sum_{i=1}^{n} u_{i} p_{i} \ln \frac{p_{i}}{A_{i}}$, which are at a minimum for the given set of constraints, simplify to

$$
\begin{gathered}
H_{1}^{*}=\left[\left(\lambda_{1}-1\right) \ln A-1-\beta\right] \tilde{P}-\left(\lambda_{1}-1-\theta\right) \tilde{A}-\alpha-\gamma \bar{P}-\tau \bar{C}-\delta \bar{A}, \\
S_{1}^{*}=\left(\lambda_{1} \ln A-1-\beta\right) \tilde{P}-\left(\lambda_{1}-\theta\right) \tilde{A}-\alpha-\gamma \bar{P}-\tau \bar{C}-\delta \bar{A} .
\end{gathered}
$$

Proof

The proof follows in the same manner as in Theorem 2.1. Consider the Lagrangian

$$
\begin{gathered}
L\left(p_{i} ; \lambda_{1}, \alpha, \beta, \gamma, \delta, \theta, \tau\right)=\sum_{i=1}^{n} u_{i} p_{i} \ln p_{i}+\left(\lambda_{1}-1\right) \sum_{i=1}^{n} u_{i} p_{i} \ln A_{i}+\alpha\left(\sum_{i=1}^{n} p_{i}-1\right) \\
+\beta\left(\sum_{i=1}^{n} u_{i} p_{i}-\tilde{P}\right)+\gamma\left(\sum_{i=1}^{n} p_{i} P_{i}-\bar{P}\right)+\delta\left(\sum_{i=1}^{n} p_{i} \ln a_{i}-\bar{A}\right) \\
+\theta\left(\sum_{i=1}^{n} u_{i} p_{i} \ln a_{i}-\tilde{A}\right)+\tau\left(\sum_{i=1}^{n} p_{i} c_{i}-\bar{C}\right) .
\end{gathered}
$$

Applying the necessary conditions of efficiency, by a direct computation, we find

$$
u_{i}+u_{i} \ln p_{i}+u_{i}\left(\lambda_{1}-1\right) \ln A_{i}+\alpha+\beta u_{i}+\gamma P_{i}+\delta \ln a_{i}+\theta u_{i} \ln a_{i}+\tau c_{i}=0,
$$

which, equivalently written, is (23). Replacing the probability in (23) into the "negative" Beliş-Guiaşu entropy $H_{1}^{*}=\sum_{i=1}^{n} u_{i} p_{i} \ln p_{i}$ and into the "negative" spatial Beliş-Guiaşu entropy $S_{1}^{*}=\sum_{i=1}^{n} u_{i} p_{i} \ln \frac{p_{i}}{A_{i}}$, by a direct computation, we obtain the "negative" measures of complexity in (24) and the proof is complete.

Further, let us consider the following notations:

$$
f_{1}(p)=\sum_{i=1}^{n} p_{i} \ln p_{i}, \quad f_{2}(p)=\sum_{i=1}^{n} p_{i} \ln p_{i}-\sum_{i=1}^{n} p_{i} \ln A_{i}
$$




$$
\begin{gathered}
h_{1}(p)=\sum_{i=1}^{n} p_{i}-1, \quad h_{2}(p)=\sum_{i=1}^{n} p_{i} P_{i}-\bar{P}, \\
h_{3}(p)=\sum_{i=1}^{n} p_{i} c_{i}-\bar{C}, \quad h_{4}(p)=\sum_{i=1}^{n} p_{i} \ln a_{i}-\bar{A} .
\end{gathered}
$$

As it can be verified, all of these functions are $(\rho, 1)$-quasiinvex at $p^{0}$, for $\rho \leq 0$ and any vector function $\theta=\theta\left(p, p^{0}\right)$ (see Definition 1.3), with respect to:

$$
\begin{aligned}
& \eta^{1}\left(p, p^{0}\right)=\left(\eta_{1}^{1}\left(p, p^{0}\right), \cdots, \eta_{n}^{1}\left(p, p^{0}\right)\right)=\left(\frac{p_{1} \ln p_{1}-p_{1}^{0} \ln p_{1}^{0}}{1+\ln p_{1}^{0}}, \cdots, \frac{p_{n} \ln p_{n}-p_{n}^{0} \ln p_{n}^{0}}{1+\ln p_{n}^{0}}\right) \\
& \eta^{2}\left(p, p^{0}\right)=\left(\eta_{1}^{2}\left(p, p^{0}\right), \cdots, \eta_{n}^{2}\left(p, p^{0}\right)\right)=\left(\frac{p_{1} \ln \frac{p_{1}}{A_{1}}-p_{1}^{0} \ln \frac{p_{1}^{0}}{A_{1}}}{1+\ln \frac{p_{1}^{0}}{A_{1}}}, \cdots, \frac{p_{n} \ln \frac{p_{n}}{A_{n}}-p_{n}^{0} \ln \frac{p_{n}^{0}}{A_{n}}}{1+\ln \frac{p_{n}^{0}}{A_{n}}}\right), \\
& \eta^{r}\left(p, p^{0}\right)=\left(\eta_{1}^{r}\left(p, p^{0}\right), \cdots, \eta_{n}^{r}\left(p, p^{0}\right)\right)=\left(p_{1}-p_{1}^{0}, \cdots, p_{n}-p_{n}^{0}\right), \quad r=3,4,5,6,
\end{aligned}
$$

where $\eta^{1}$ is the vector function associated with $f_{1}, \eta^{2}$ is the vector function associated with $f_{2}, \eta^{3}$ is the vector function associated with $h_{1}$, and so on.

The following result formulates some sufficient conditions of efficiency for our vector minimization problem $(V O P)$.

\section{Theorem 2.3}

Let $p^{0}=\left(p_{i}^{0}\right), i=\overline{1, n}$, be any feasible solution to $(V O P)$ and let there exist the scalars $\lambda_{1}, \lambda_{2}, \alpha, \beta, \gamma, \delta$ with $\lambda_{1}>0, \lambda_{2}>0, \lambda_{1}+\lambda_{2}=1$, satisfying

$$
p_{i}^{0}=\exp \left[-1-\alpha-\left(1-\lambda_{1}\right) \ln A-\beta P_{i}-\gamma c_{i}-\left(\lambda_{1}+\delta-1\right) \ln a_{i}\right] .
$$

If at least one of the functions defined in $(25)$ is strictly $(\rho, b)$-quasiinvex at $p^{0}$ with respect to $\eta$ and $\theta$, and

$$
\lambda_{1} \rho_{1}^{1}+\lambda_{2} \rho_{2}^{1}+\alpha \rho_{1}^{3}+\beta \rho_{2}^{3}+\gamma \rho_{3}^{3}+\delta \rho_{4}^{3} \geq 0,
$$

then $p^{0}$ is an efficient solution to $(V O P)$.

\section{Proof}

First, we have to mention that the real numbers $\rho_{i}^{1}, i=1,2$, and $\rho_{k}^{3}, k=\overline{1,4}$, introduced in our theorem, have the same siqnificance as in Remark 1.4 of section 1.

As we have previously established, the functions given in $(25)$ are $(\rho, 1)$-quasiinvex at $p^{0}$, for $\rho \leq 0$ and any vector function $\theta=\theta\left(p, p^{0}\right)$, with respect to the vector functions $\eta=\eta\left(p, p^{0}\right)$ formulated in (26), respectively. As well, we notice that, if we consider $\rho<0$ and $\theta \neq \overline{0}$, then we obtain strictly $(\rho, 1)$-quasiinvexity at $p^{0}$ with respect to $\eta$ and $\theta$ for our functions in (25). Consequently, we have at least one function in (25) which is strictly $(\rho, b)$-quasiinvex at $p^{0}$ with respect to $\eta$ and $\theta$.

Further, taking into account Theorem 1.2 and Remark 1.4 of section 1 (see conditions $(i i i)$ and $\left(i i i^{\prime}\right)$ ), the proof is complete.

In a similar way, one can find a characterization result of sufficient conditions regarding the weighted bi-objective optimization problem $(V O P)^{*}$.

As it can be verified, all of the following functions

$$
\begin{gathered}
f_{1}(p)=\sum_{i=1}^{n} u_{i} p_{i} \ln p_{i}, \quad f_{2}(p)=\sum_{i=1}^{n} u_{i} p_{i} \ln p_{i}-\sum_{i=1}^{n} u_{i} p_{i} \ln A_{i} \\
h_{1}(p)=\sum_{i=1}^{n} p_{i}-1, \quad h_{2}(p)=\sum_{i=1}^{n} u_{i} p_{i}-\tilde{P}
\end{gathered}
$$




$$
\begin{gathered}
h_{3}(p)=\sum_{i=1}^{n} p_{i} P_{i}-\bar{P}, \quad h_{4}(p)=\sum_{i=1}^{n} p_{i} \ln a_{i}-\bar{A}, \\
h_{5}(p)=\sum_{i=1}^{n} u_{i} p_{i} \ln a_{i}-\tilde{A}, \quad h_{6}(p)=\sum_{i=1}^{n} p_{i} c_{i}-\bar{C},
\end{gathered}
$$

are $(\rho, 1)$-quasiinvex at $p^{0}$, for $\rho \leq 0$ and any vector function $\theta=\theta\left(p, p^{0}\right)$, with respect to

$$
\begin{gathered}
\eta^{1}\left(p, p^{0}\right)=\left(\eta_{1}^{1}\left(p, p^{0}\right), \cdots, \eta_{n}^{1}\left(p, p^{0}\right)\right)=\left(\frac{p_{1} \ln p_{1}-p_{1}^{0} \ln p_{1}^{0}}{1+\ln p_{1}^{0}}, \cdots, \frac{p_{n} \ln p_{n}-p_{n}^{0} \ln p_{n}^{0}}{1+\ln p_{n}^{0}}\right), \\
\eta^{2}\left(p, p^{0}\right)=\left(\eta_{1}^{2}\left(p, p^{0}\right), \cdots, \eta_{n}^{2}\left(p, p^{0}\right)\right)=\left(\frac{p_{1} \ln \frac{p_{1}}{A_{1}}-p_{1}^{0} \ln \frac{p_{1}^{0}}{A_{1}}}{1+\ln \frac{p_{1}^{0}}{A_{1}}}, \cdots, \frac{p_{n} \ln \frac{p_{n}}{A_{n}}-p_{n}^{0} \ln \frac{p_{n}^{0}}{A_{n}}}{1+\ln \frac{p_{n}^{0}}{A_{n}}}\right), \\
\eta^{r}\left(p, p^{0}\right)=\left(\eta_{1}^{r}\left(p, p^{0}\right), \cdots, \eta_{n}^{r}\left(p, p^{0}\right)\right)=\left(p_{1}-p_{1}^{0}, \cdots, p_{n}-p_{n}^{0}\right), \quad r=\overline{3,8},
\end{gathered}
$$

respectively. Also, if we consider $\rho<0$ and $\theta \neq \overline{0}$, then we obtain strict $(\rho, 1)$-quasiinvexity at $p^{0}$ with respect to $\eta$ and $\theta$ for our functions in (27).

Theorem 2.4

Let $p^{0}=\left(p_{i}^{0}\right), i=\overline{1, n}$, be any feasible solution to $(V O P)^{*}$ and let there exist the scalars $\lambda_{1}, \lambda_{2}, \alpha, \beta, \gamma, \delta, \theta$ and $\tau$, with $\lambda_{1}, \lambda_{2}>0, \lambda_{1}+\lambda_{2}=1$, satisfying

$$
p_{i}^{0}=\exp \left[-u_{i}-u_{i}\left(\lambda_{1}-1+\theta\right) \ln a_{i}-u_{i}\left(1-\lambda_{1}\right) \ln A-\alpha-\beta u_{i}-\gamma P_{i}-\delta \ln a_{i}-\tau c_{i}\right] / u_{i} .
$$

If at least one of the functions defined in (27) is strictly $(\rho, b)$-quasiinvex at $p^{0}$ with respect to $\eta$ and $\theta$, and

$$
\lambda_{1} \rho_{1}^{1}+\lambda_{2} \rho_{2}^{1}+\alpha \rho_{1}^{3}+\beta \rho_{2}^{3}+\gamma \rho_{3}^{3}+\delta \rho_{4}^{3}+\theta \rho_{5}^{3}+\tau \rho_{6}^{3} \geq 0,
$$

then $p^{0}$ is an efficient solution to $(V O P)^{*}$.

Proof

The proof follows in the same manner as in Theorem 2.3.

Remark 2.2

i) If we consider $A_{i}=a_{i}, i=\overline{1, n}$, (see section 1) in the bi-objective minimization problem $(V O P)$, then we get

$$
\begin{gathered}
\min _{p_{i}}\left(\sum_{i=1}^{n} p_{i} \ln p_{i}, \quad \sum_{i=1}^{n} p_{i} \ln p_{i}-\bar{A}\right) \\
\text { subject to } \\
\sum_{i=1}^{n} p_{i}=1, \quad \sum_{i=1}^{n} p_{i} P_{i}=\bar{P}, \quad \sum_{i=1}^{n} p_{i} c_{i}=\bar{C},
\end{gathered}
$$

where we used the constraint $\sum_{i=1}^{n} p_{i} \ln a_{i}=\sum_{i=1}^{n} p_{i} \ln A_{i}=\bar{A}$. Therefore, our constrained multi-objective optimization problem $(V O P)$ become a single-objective optimization problem because the two objective functions differ by a constant.

ii) If we take $A_{i}=a_{i}, i=\overline{1, n}$, and remove the constraint $\sum_{i=1}^{n} p_{i} \ln a_{i}=\bar{A}$ in the minimization problem $(V O P)$, then we obtain

$$
\min _{p_{i}}\left(\sum_{i=1}^{n} p_{i} \ln p_{i}, \quad \sum_{i=1}^{n} p_{i} \ln p_{i}-\sum_{i=1}^{n} p_{i} \ln a_{i}\right)
$$


subject to

$$
\sum_{i=1}^{n} p_{i}=1, \quad \sum_{i=1}^{n} p_{i} P_{i}=\bar{P}, \quad \sum_{i=1}^{n} p_{i} c_{i}=\bar{C},
$$

a freestanding constrained bi-objective minimization problem. By imposing the necessary conditions of efficiency, we get

$$
\begin{aligned}
p_{i} & =\exp \left[-1-\alpha-\beta P_{i}-\gamma c_{i}-\left(\lambda_{1}-1\right) \ln a_{i}\right] \\
& =K \exp \left[-\beta P_{i}-\gamma c_{i}-\left(\lambda_{1}-1\right) \ln a_{i}\right],
\end{aligned}
$$

where $K=\exp [-1-\alpha]$ is a constant of proportionality which ensures that the probabilities sum is 1 . The associated "negative" measures of complexity $H_{1}=-H=\sum_{i=1}^{n} p_{i} \ln p_{i}$ and $S_{1}=-S=\sum_{i=1}^{n} p_{i} \ln \frac{p_{i}}{a_{i}}$, which are at a minimum for the given set of constraints, simplify to

$$
\begin{gathered}
H_{1}=-1-\alpha-\beta \bar{P}-\gamma \bar{C}-\left(\lambda_{1}-1\right) \sum_{i=1}^{n} p_{i} \ln a_{i}, \\
S_{1}=-1-\alpha-\beta \bar{P}-\gamma \bar{C}-\lambda_{1} \sum_{i=1}^{n} p_{i} \ln a_{i} .
\end{gathered}
$$

Also, let us notice that the assumption $A_{i}=a_{i}, i=\overline{1, n}$, ensures that the fraction $\frac{p_{i}}{A_{i}}$ always remains subunitary. iii) Let us consider the second objective function in $(V O P), f_{2}(p)=\sum_{i=1}^{n} p_{i} \ln p_{i}-\sum_{i=1}^{n} p_{i} \ln A_{i}$. Taking into account the constraints $\sum_{i=1}^{n} p_{i} \ln a_{i}=\bar{A}, \sum_{i=1}^{n} p_{i}=1$ and the relation $A_{i}=\frac{a_{i}}{A}, i=\overline{1, n}$ (see section 1 ), we find

$$
\begin{gathered}
f_{2}(p)=\sum_{i=1}^{n} p_{i} \ln p_{i}-\sum_{i=1}^{n} p_{i} \ln a_{i}+\sum_{i=1}^{n} p_{i} \ln A \\
=f_{1}(p)-\bar{A}+\ln A=f_{1}(p)+\text { const. }
\end{gathered}
$$

that is, the two objective functions differ by a constant and $(V O P)$ become a single-objective optimization problem. Consequently, the general case is obtained when we consider $A_{i}=a_{i}, i=\overline{1, n}$, and remove the constraint $\sum_{i=1}^{n} p_{i} \ln a_{i}=\bar{A}$ in the minimization problem $(V O P)$ (see ii)).

iv) Similar remarks as above can be formulated for $(V O P)^{*}$.

\section{Conclusions}

In the present work, using the classical multiobjective optimization theory, we have investigated a simultaneous optimization of the information measures given in (7) and (8) and of the weighted measures of information given by Beliş-Guiaşu entropy $H^{*}=-\sum_{i=1}^{n} u_{i} p_{i} \ln p_{i}$ and spatial Beliş-Guiaşu entropy $S^{*}=-\sum_{i=1}^{n} u_{i} p_{i} \ln \frac{p_{i}}{A_{i}}$. However, what is of more significant here is the kind of distribution that we have obtained. Practically, we have derived an approximation on the distribution of population densities and the arrangement of urban activities over a set of $n$ locations. For other different ideas but connected to this topic (information theory), the reader is directed to Wilson [19], Preda [13], Anas [1], Halliwell and Mercer [8], Liese and Vajda [11], Srivastava [15]. 


\section{Acknowledgement}

This work has been funded by University Politehnica of Bucharest, through the "Excellence Research Grants" Program, UPB - GEX. Identifier: UPB-EXCELENŢĂ-2016 Matematici Aplicate în Urbanism (Applied Mathematics in Urbanism), Contract number 25/26.09.2016.

\section{REFERENCES}

1. A. Anas, Discrete choice theory, information theory and the multinomial logit and gravity models, Transp. Res. B. 17(1983), no. 1, 13-23.

2. M. Arana-Jiménez, G. Ruiz-Garzón, R. Osuna-Gómez and B. Hernández-Jiménez, Duality and a characterization of pseudoinvexity for Pareto and weak Pareto solutions in nondifferentiable multiobjective programming, J. Optim. Theory Appl. 156(2013), no. 2, 266-277.

3. M. Batty, Spatial entropy, Geograph. Anal. 6(1974), no. 1, 1-31.

4. M. Batty, Cities as complex systems: Scaling, interactions, networks, dynamics and urban morphologies. In R. Meyers (Ed.). Encyclopedia of complexity and systems science (Vol. 1, pp. 1041-1071). Berlin, DE: Springer, 2009.

5. M. Batty, R. Morphet, P. Masucci and K. Stanilov, Entropy, complexity, and spatial information, J. Geogr. Syst. 16(2014), no. 4, 363-385.

6. M. Beliş and S. Guiaşu, A Quantitative-Qualitative Measure of Information in Cybernetic Systems, IEEE Transactions on Information Theory 14(1968), no. 4, 593-594.

7. R. Clausius, Ueber verschiedene für die Anwendung bequeme formen der Hauptgleichungen der mechanischen Wärmetheorie, Ann. Phys. Chem. 125(1865), 53-400.

8. G.T. Halliwell and P.R. Mercer, A Refinement of an Inequality from Information Theory, Journal of Inequalities in Pure and Applied Mathematics 5(2003), no. 3, 29-31.

9. J.N. Kapur, On the Concept of "Useful" Information, J. Org. Behav. Stat. 2(1985), no. 3-4, 147-162.

10. J.N. Kapur, New Qualitative-Quantitative Measures of Information, Nat. Acad. Sci. Lett. 9(1986), no. 2, 51-54.

11. F. Liese and I. Vajda, On divergences and informations in statistics and information theory, IEEE Transactions on Information Theory 52(2006), no. 10, 4394-4412.

12. G. Longo, Qualitative-Quantitative Measures of Information, New York, Springer-Verlag, 1972.

13. V. Preda, The Student distribution and the principle of maximum entropy, Ann. Inst. Statist. Math. A 34(1982), no. 1, $335-338$.

14. C.E. Shannon, A Mathematical Theory of Communication, Bell. Syst. Tech. Jour., 2(1948), 623-659.

15. A. Srivastava, Some New Bounds of Weighted Entropy Measures, Cybernetics and Information Technologies 11(2011), no. 3, 60-65.

16. S. Treanţă and C. Udrişte, On efficiency conditions for multiobjective variational problems involving higher order derivatives, Proceedings of the 15th International Conference on Automatic Control, Modelling \& Simulation (ACMOS'13), 157-164, 2013.

17. S. Treanţă, Multiobjective fractional variational problem on higher-order jet bundles, Commun. Math. Stat. 4(2016), no. 3, 323-340.

18. R.U. Verma, Mathematical programming based on sufficient optimality conditions and higher order exponential type generalized invexities, Stat., Optim. Inf. Comput. 3(2015), no. 3, 276-293.

19. A.G. Wilson, Entropy in urban and regional modelling, Pion Press, London, 1970.

20. P.L. Yu, Multi-Criteria Decision Making: Concepts, Techniques, and Extensions, Plenum Press, New York, 1985. 